\title{
Angiomixoma cutáneo primario
}

Primary cutaneous angiomyxoma

Moretti, A.*, Gallo, S.**, Savoré, M.***, Monti, J .****

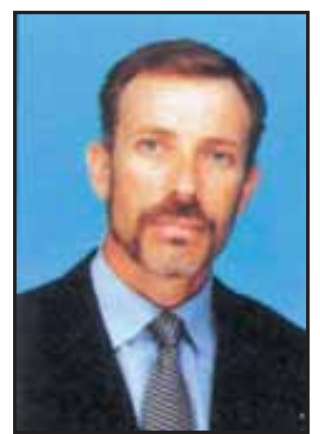

Moretti, A.
Resumen

Se describe el caso de un paciente de 50 años de edad con angiomixoma cutáneo, sin evidencia del complejo de Carney y con compromiso de la región témporo-frontal. Dicho tumor cutáneo poseía el diagnóstico ecográfico erróneo de lipoma. El tratamiento fue la exéresis amplia; no se ha evidenciado recurrencia tras 4 años de seguimiento. Se describe el caso clínico debido a la rareza de la patología y a la confusión con otros tumores del tejido celular subcutáneo. Revisamos también los estudios histopatológicos y diagnósticos diferenciales, así como la terapéutica y los índices de recidiva.
Palabras clave Tumor subcutáneo. Mixomas

Código numérico 175
Key words Subcutaneous tumour. Myxomas

Numeral Code 175

\footnotetext{
* J efe del Servicio de Cirugía Plástica, Sanatorio Los Arroyos.

* Médico Residente.

* Médica Dermatóloga.

* Méck Médico Anatomo-patólogo.
}

Servicio de Cirugía Plástica, Sanatorio Los Arroyos. Rosario, República Argentina.

We present a 50 years old patient with a cutaneous angiomixoma without evidence of Carney's complex that affected the temporo-frontal region. This tumour had an echography misdiagnosis of lipoma. The treatment was exeresis with safe margins. There was no evidence of recurrence of the tumour in a 4 year follow up. Due to the uncommonness of the pathology, we describe this clinical case, histopathology studies, differential diagnosis, as well as therapeutic and recurrence indexes. 


\section{Introducción}

Los mixomas cutáneos solitarios, llamados también angiomixomas, para enfatizar su componente vascular, son una neoplasia benigna dérmica y subcutánea, relativamente poco común, generalmente solitarios, con un alto índice de recidiva pero sin potencial metastático (1).

Estos tumores generalmente se presentan como un nódulo de crecimiento lento y asintomático, localizado más frecuentemente en músculo esquelético y cardíaco, maxilares (mixoma odontogénico), pero también pueden observarse en hueso, intestino, conjuntiva y tejido celular subcutáneo de cabeza, cuello y tronco de adultos $(2,3)$ en la segunda o tercera década de vida (rango entre 20 y 40 años).

El término mixomas cutáneos solitarios se utiliza para diferenciarlos de los complejos de mixomas (cardíacos, cutáneos y mamarios), pigmentación moteada (cutánea o mucocutánea), e hiperactividad endocrina (síndrome de Cushing, tumor testicular, precocidad sexual y acromegalia) descripto por el Dr. Carney y colaboradores $(4,5)$ A los mixonas cutáneos también se los ha asociado con el síndrome N.A.M.E. (6) (Nevus, Mixoma auricular, Neuromixoma mixoide y Sobreactividad endocrina) y L.A.M.B. (7-8) (Lentiginosis, Mixoma auricular, Mixoma cutáneo y Nevus azules).

La condición que le confiere el sufijo "oma" implica su separación de todos aquellos depósitos de sustancia (mucinosis) cuyo carácter localizado les confiere aspecto seudotumoral (ganglión o mixoma sinovial, mucinosis cutánea focal, quiste mucoso digital, etc.).

Los mixomas cutáneos solitarios fueron descritos como tumores vinculados al folículo piloso (3) o como "nódulos blandos y lobulados que sobreelevan la piel en diferente grado" (2). A través de los años, solo unos pocos casos de mixomas cutáneos solitarios sin características sistémicas han sido referidos en la literatura (1,3-9).

\section{CASO CLINICO}

Se describe el caso de un paciente de 50 años de edad, derivado desde el Servicio de Dermatología por presentar un tumor subcutáneo en la región témporoparietal derecha de 2 años de evolución, (Fig. 1) con crecimiento lento, asintomático y con estudio ultrasonográfico preoperatorio de lesión lipomatosa. No hay referencia de antecedentes familiares de lesión similar, traumatismo u otra característica indicativa de naturaleza sindrómica. Se procedió a la exéresis quirúrgica de la lesión bajo anestesia local y se constató un tumor de $3 \times 3 \mathrm{~cm}$ con contenido gelatinoso transparente y sin evidencia de cápsula (Fig. 2). El informe

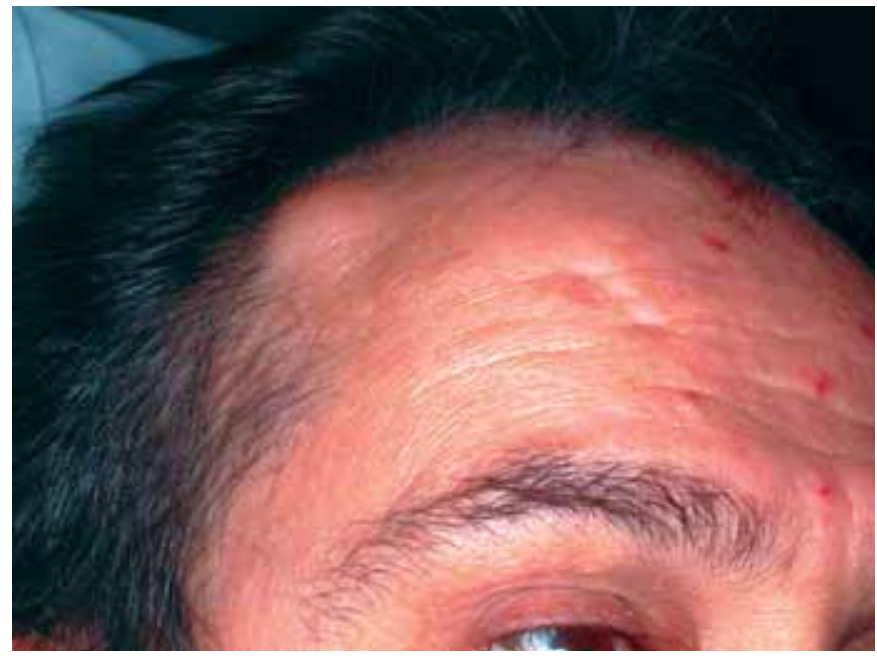

Fig. 1. Presentación caso. Tumor en región temporo-frontal.

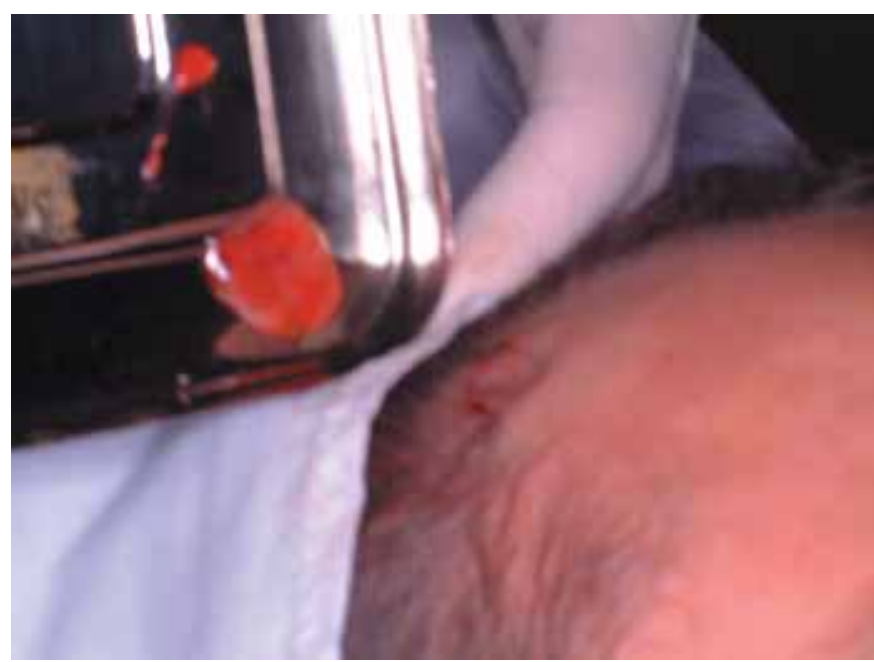

Fig. 2. Exéresis mostrando tumor gelatinoso y sin evidencia de cápsula.

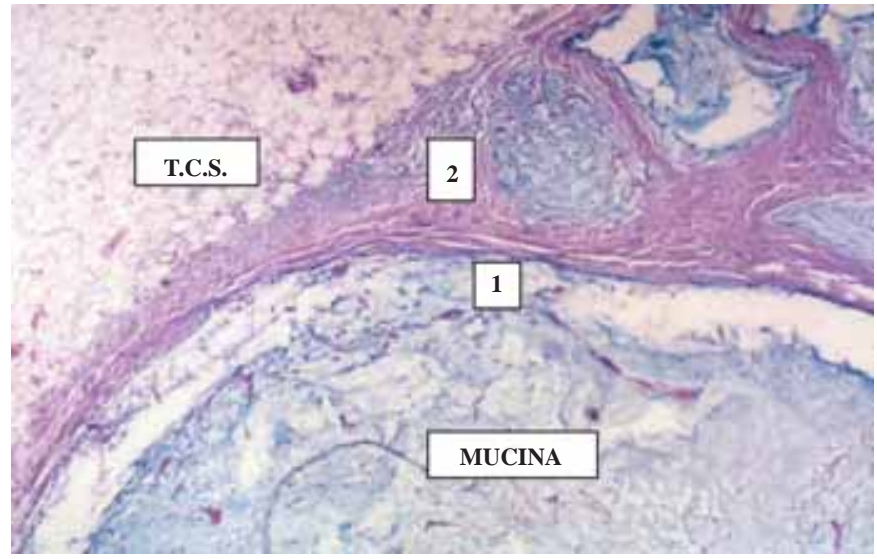

Fig. 3. Coloración: Alzacyan Blue 100x. 1. Tejido angiomixoide. 2. Aparente "cápsula" Azul: mucina. Blanco: TCS

intraoperatorio de la muestra fue de mixoma cutáneo, decidiendo en ese momento el cambio de plan quirúrgico para proceder a la ampliación de los márgenes de extirpación de seguridad a $2 \mathrm{~cm}$. La evolución postoperatoria fue satisfactoria y el paciente se ha mantenido sin evidencia de recidiva en los 4 años de seguimiento. 


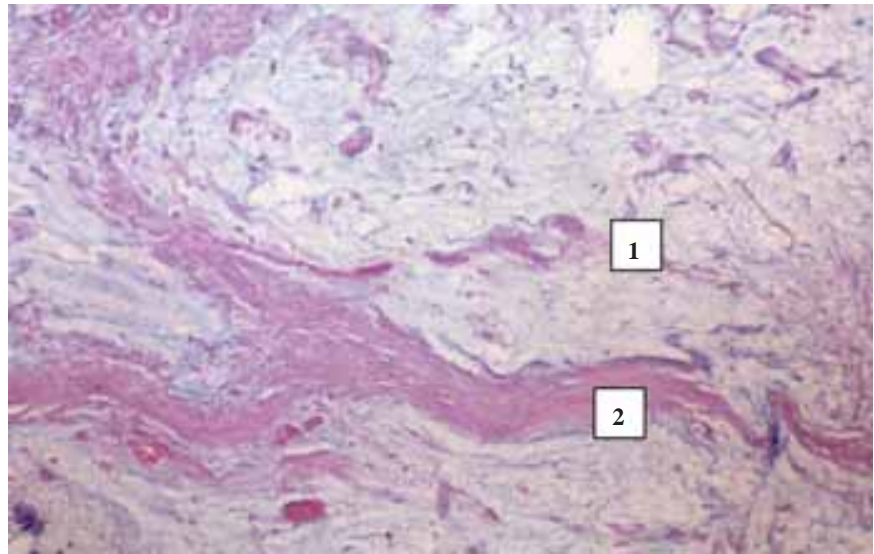

Fig. 4. Coloración: Alzacyan Blue 100x. 1. Proliferación vascular ramificada. 2. Septums de tejido fibroso (tej. esclerohialino denso).

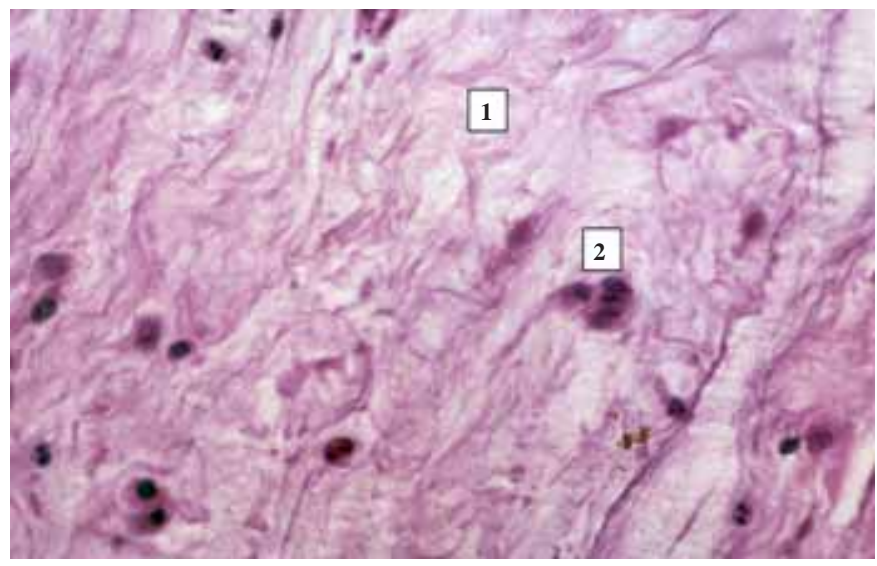

Fig. 5. Coloración: H \& E 400x. 1. Matriz mixoide laxa fibrilar. 2. Ocasionales plasmocitos.

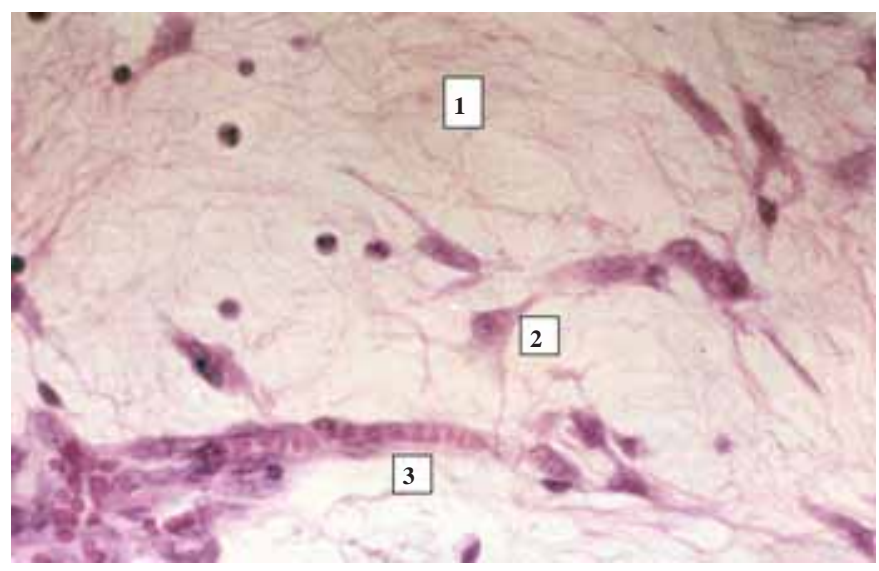

Fig. 6. Coloración: H \& E 400x. 1. Matriz mixoide. 2. Células fibroblásticas estelares. 3. Vaso sanguíneo (corte long).

\section{Histopatología}

Desde el punto de vista anatomopatológico, la formación conformaba una masa ovoide bien delimitada periféricamente, muy friable y gelatinosa, que a la observación microscópica se componía de extensos lagos o acúmulos de material mucoso, azul alzacian positivo a $\mathrm{pH}$ bajo, sobre un lecho de estructuras vasculares proliferantes, ramificadas, sin atipias citológicas y escasos fibroblastos estelares. El estroma era marcadamente hipocelular, mixoide, y con contenido de mucopolisacáridos. Dentro del estroma mixoide basófilo, se encontraron pequeñas células con núcleos ovales y citoplasma mal definido. Se observó vascularización abundante así como lobulaciones y elementos epiteliales (estos elementos epiteliales serían residuos de estructuras anexiales) (2). Pese al neto contorno del proceso no se constató cápsula verdadera. No se observó hipercromia, atipia nuclear, presencia de adipocitos o lipoblastos o anomalías madurativas del componente vascular, siendo el diagnóstico final de angiomixoma cutáneo (Fig. 3-6).

\section{Discusión}

Los mixomas cutáneos solitarios se localizan en la dermis o en el tejido celular subcutáneo; están perfectamente circunscritos sin encapsulación cuando se observan en la dermis pero ocasionalmente circunscritos cuando se encuentran en el tejido graso subcutáneo. El estroma es marcadamente hipocelular, mixoide, y contiene mucopolisacáridos. Dentro del estroma mixoide basófilo, hay pequeñas células fusadas y estelares con núcleos ovales o lanceolados y citoplasma mal definido que expresan positividad para vimentina. Se puede observar vascularización abundante, ramificada, así como lobulaciones. La presencia de elementos epiteliales se debe al atrapamiento de restos anexiales. Un carácter proliferativo de los mismos debe inducir a pensar en otro tipo de neoplasias asociadas a mucinas de origen epitelial, derivadas de la vaina externa del folículo piloso (tricoblastomas, mixoma folicular, etc.) o de células epiteliales ecrinas (siringoma condroide o tumor mixto de la piel) (2-10). Las mucinas presentes en este tipo de tumores, son de origen fibroblástico, a diferencia de la de origen epitelial. La coloración de azul alzacian a pH bajo, es especifica de la misma.

La localización de estos tumores en la cabeza y el cuello corresponde al $17 \%$ del total de la patología $(11,12)$.

Los mixomas cutáneos múltiples, son generalmente correlacionados con el complejo de Carney (4), síndrome N.A.M.E. (6) o L.A.M.B. (7). Los mixomas que se observan en el complejo de Carney, generalmente afectan a pacientes jóvenes, y usualmente son de menos de $1 \mathrm{~cm}$. de tamaño. Son multicéntricos y tienen una distribución diseminada, con predilección por párpados, orejas y pezones $(13,14)$. Son frecuentemente descritos como pápulas sésiles o nódulos subcutáneos, asintomáticos, de superficie lisa sin ulceraciones; raramente son papilomatosos y de superficie áspera (4). Estos Mixomas usualmente notifican la presencia de un tumor cardíaco y a pesar 
de su naturaleza benigna, tienen tendencia a la recidiva local, posterior a la escisión.

Los Mixomas solitarios, sin otras manifestaciones del complejo de Carney, generalmente crecen en el músculo esquelético o cardíaco, con menor frecuencia en el intestino, pelvis, tejido celular subcutáneo (15) y extraordinariamente en la piel (16). Otras referencias de la literatura (17-19) describen lesiones únicas o múltiples categorizadas como mucinosis cutánea focal, no siendo consideradas como verdaderos Mixomas. Se caracterizan por ser una acumulación local de material mucinoso en la dermis, con "scattered spindle cells" pero con menor vascularización que los Mixomas (11).

Los mixomas cutáneos deber ser diferenciados de neoplasias de partes blandas que secundariamente producen mucinas como los tumores neurales (neurofibromas, neurotekeomas, etc.), fibroblásticos (tumores fibromixoides osificantes y no osificantes) o adiposos (liposarcoma mixoide).

Con respecto a la terapéutica, la única opción es la exéresis quirúrgica amplia con buenos márgenes de seguridad aunque hay que tener en cuenta que los ubicados en región pelviana, perineal y vulvar poseen un carácter muy agresivo local y recidivante (20). Los estudios retrospectivos en la literatura, marcan la diferenciación entre los angiomixomas agresivos (recurrentes, infiltrantes) de la región pelvicoperineal y los no agresivos de otras regiones. Aún hoy se debate al respecto de si se trata de distintas variedades de un mismo tumor, ya que sus diferencias clínicas y patológicas serían debidas principalmente a los diferentes estadíos de su diagnóstico.

La recurrencia local de los tumores solitarios del tejido celular subcutáneo está en torno al $38 \%$ de los casos (12), de modo que fueron considerados como tumores de comportamiento agresivo; es de destacar que en los angiomixomas con componente epitelial se observa una recurrencia mayor $(63 \%)(12,15,20)$ a la observada en estos mismos sin ese componente $(23 \%)(7,12,21)$. Este comportamiento parece ser el resultado de la localización preferentemente subcutánea de este tipo de lesiones. Por tal motivo es de resaltar la importancia de la resección quirúrgica profunda y amplia con un el examen físico detallado y ultrasonido cardiológico, para descartar el complejo de Carney.

\section{Conclusiones}

Se presenta un caso de angiomixoma cutáneo solitario de ubicación en la región témporo-frontal derecha con diagnóstico previo de lipoma subcutáneo. El tratamiento es la exéresis amplia con márgenes de seguridad de $2 \mathrm{~cm}$. Estos tumores poseen posibilida- des de recidiva de entre el 23 y $63 \%$ dependiendo de la presencia del componente epitelial dentro del estudio histopatológico.

\section{Dirección del autor}

\author{
Dr. Ernesto A. Moretti \\ Italia 1460 \\ 2000 Rosario. República Argentina \\ e-mail: doctormoretti@knett.com.az
}

\section{Bibliografía}

1. Alaiti S, Nelson FP, Ryoo JW. "Solitary cutaneous myxoma". J Am Acad Dermatol. 2000; 43:377.

2. Allen PW, Dymock RB, MacCormac LB. "Superficial angiomyxomas with and epithelial components". Am J Pathol 1988; 12:519.

3. Wilk M, Schmoeckel C, Kaiser HW, Hepple R, Kreysel HW. "Cutaneous angiomyxoma; a benign neoplasm distinct from cutaneous focal mucinosis". Am Acad Dermatol. 1995; ; 33:352.

4. Carney JA, Gordon H, carpenter PC, Shenoy BV, Go VW. "The complex of myxomas, spotty pigmentation, and endocrine overactiviti." Medicine (Balt) 1985;64:270.

5. Takahashi H, Hida T. "Carney complex: report of a Japanese case associated with cutaneous superficial angiomyxomas, labial lentigines, and a pituitary adenoma". J Dermatol. 2002 ; 29(12):790.

6. Atherton DJ, Pitcher DW, Well RS, MacDonald DM. "A syndrome of varius cutaneus pigmented lesion, myxoid neurofibromata and atrial myxomas: the name syndrome". Br. J Dermatol 1980;103:421.

7. Rhodes AR, Silverman RA, Harrist TJ, Perez-Atayde AR. "Mucocutaneus lentigines, cardiomucocutaneus and multiple blue nevi; the "LAMB" syndrome. J Am Acad Dermatol 1984;10:72.

8. Egan CA, Stratakis CA, Turner ML. "Multiple lentigines associated with cutaneous myxomas". J Am Acad Dermatol. 2001; 44(2):282

9. Murphy CM, Grau-Massanares M, Sanches RL.: "Multiple cutaneus myxomas: report of a case without other elements of carmey`s complex". J cutan Pathol 1995;22:556.

10. Enzinger FM, Weiss SW, editors. "Soft tissue tumors", $3^{\text {rd }}$ ed. St. louis; CV Mosby; 1998.

11. Wilk M, Schmokel C. "Cutaneous focal mucinosis: a histophatological and immunohistochemical analysis of 11 cases". J Cutan Pathol 1994;21:446.

12. Allen PW, Dymock RB, MacCormac LB. "Superficial angiomyxomas with and without epithelial components". Am J Surg Pathol. $1988 ; 12(7): 519$

13. Yuen HK, Cheuk W, Luk FO, Wat CS, Auyeung KC, Lam DS. "Solitary superficial angiomyxoma in the eyelid". Am J Ophthalmol. $2005 ; 139(6): 1141$

14. Grossniklaus HE, McLean IW, Gillespie JJ. "Bilateral eyelid myxomas in Carney’s complex". Br J Ophthalmol. 1991; 75 (4): 251

15. Stout Ap. "Myxoma, the tumor of primitive mesenchyme". Ann Surg 1948;127:706

16. Dutz W, stout AP. "The myxoma in childhood". Cancer 1961; $14: 629$

17. Johson WC, Helwig EB. "Cutaneus focal mucinosis: a clinicopathological and histochemical study". Arch Dermatol 1966;93:13.

18. Suhomen R, Neimi KM. "Cutaneus focal mucinosis with spontaneous healing". J Cutan Pathol 1983;10:334.

19. Jakubovic HR, Salama SS, Rosenthal D. "Multiple cutaneous focal mucinosis with hypothyroidism". Ann Int Med 1982;96:56

20. Argenyi ZB, Leboit PE, Santa Cruz D, Swanson PE, Kutzner H. "Nerve sheath myxoma (neurothekeoma) of the skin; light microscopic and immunohistochemical reappraisal of the celular variant" J Cutan Pathol 1993;20:294.

21. Senff H, Kuhlwein A, Jänner M, et al. "Kutanes Myxom (fokale dermale Muzinose)". Hautarzt 1988;39:606 